\title{
VACUNA EMOCIONAL EN LA COVID-19: PROGRAMA DE AUTOCUIDADO PARA PERSONAS MAYORES
}

\author{
Marta Florencia Abril Herrero' \\ Colegio Oficial de Psicología de Castilla y León \\ Grupo de Trabajo de Psicología del Envejecimiento \\ Valladolid \\ dcleon@cop.es \\ María Elvira Zorzo ${ }^{1}$ \\ María Yolanda González Alonso' \\ Estefanía González Arranz ${ }^{1}$ \\ Vanessa Hernández Calvo ${ }^{1}$ \\ Carolina Pastoriza Serradilla' \\ Ana Isabel Rueda Lozano' \\ Ana Belén Sánchez Sánchez ${ }^{1}$ \\ Soledad Tundidor Gago'
}

Recepción Artículo: 15 mayo 2021 Admisión Evaluación: 15 mayo 2021 Informe Evaluador 1: 19 mayo 2021 Informe Evaluador 2: 24 mayo 2021 Aprobación Publicación: 01 junio 2021

\section{RESUMEN}

El colectivo de personas mayores es uno de los más afectados por esta pandemia. Es considerado grupo de riesgo y la edad es un factor relevante en la respuesta que da el organismo frente a la Covid-19. Para afrontar esta situación y esta enfermedad es importante que las personas se cuiden a nivel biopsicosocial. El presente trabajo tiene como fin exponer un programa de autocuidado y de activación psicológica: "Vacuna Emocional para la COVID-19". Dirigido a personas mayores sin o con deterioro cognitivo leve, de manera individual o grupal, en diferentes entornos: residencial, domiciliario, centro de día, etc., valorando el efecto de este programa en la mejora de su calidad de vida, distinguiendo entre contextos rurales y urbanos. El programa consta de 10 sesiones de una hora y media de duración aplicándose dos sesiones semanales. En cada sesión se trabaja una pauta de autocuidado a través de un mensaje con forma de refrán que facilita la activación mnésica. Desde la Terapia de Aceptación y Compromiso y siguiendo el paradigma de la Atención Centrada en lo Importante para la Persona, se aborda la gestión emocional, la estimulación cognitiva y la activación conductual de manera que se produzca un mejor afrontamiento de la situación actual. Se enfatiza el proyecto de vida de cada participante a través de la orientación en valores. Utilizando un diseño cuasiexperimental pre-post con grupo control, se emplean los instrumentos: registros conductuales, escalas y cuestionarios que midan calidad de vida, ansiedad, depresión y evitación experiencial. Se espera una mejora en la salud y bienestar a través de la activación psicológica en la mues- 
tra diana respecto al grupo control. Se destaca la necesidad de este tipo de intervención psicológica en el ámbito gerontológico por su viabilidad, eficacia y demanda social.

Palabras clave: aceptación y compromiso; activación conductual; autocuidado; COVID-19; envejecimiento; intervención psicológica

\begin{abstract}
Emotional vaccine at covid-19: self-care program for seniors. The group of elderly people is one of the most affected by this pandemic. It is considered a risk group and age is a relevant factor in the body's response to COVID-19. To face this situation and disease, it is important that people take care of themselves at a biopsychosocial level. The aim of this paper is to present a self-care and psychological activation program: "Emotional Vaccine for COVID-19". It is aimed at elderly people without or with mild cognitive impairment, individually or in groups, in different environments: residential, home, day center, etc., assessing the effect of this program in the improvement of their quality of life, distinguishing between rural and urban contexts. The program consists of 10 sessions of one hour and a half, held twice a week. In each session, a self-care guideline is worked on through a message in the form of a saying that facilitates mnesic activation. From the Acceptance and Commitment Therapy and following the paradigm of the Attention Centered on what is Important for the Person, emotional management, cognitive stimulation and behavioral activation are addressed in order to produce better coping strategies for the current situation. The life project of each participant is emphasized through orientation to values. Using a quasi-experimental pre-post design with a control group, the following instruments are used: behavioral records, scales and questionnaires that measure quality of life, anxiety, depression and experiential avoidance. An improvement in health and well-being through psychological activation is expected in the target sample with respect to the control group. The need for this type of psychological intervention in the gerontological field is highlighted due to its feasibility, efficacy and social demand.
\end{abstract}

Keywords: acceptance and commitment; behavioral activation; self-care; COVID-19; aging; psychological intervention

\title{
ANTECEDENTES
}

El aumento de personas mayores es uno de los cambios más significativos que se ha dado en las sociedades desarrolladas a lo largo de la segunda mitad del siglo XX (Lorenzo, 2004) y actualmente este colectivo continúa en proceso de envejecimiento (Abellán et al., 2019). Imserso (2009), indica que en los países industrializados existe un aumento progresivo del número absoluto de personas mayores, siendo España uno de los países que presenta una las situaciones más críticas, puesto que la población mayor de 65 años ya representaba en el año 2012 el 16,9\% de la población total y esta proporción se calcula que aumentará paulatinamente hasta llegar al $36,8 \%$ en el 2049 , el 18,2\% serán mayores de 75 años, y el 11,8\% serán mayores de 80 años, esto significa que una de cada tres mayores tendrán más de 80 años (Figura 1), mostrando los datos una mayor expectativa de vida en las mujeres (Rodríguez, 2021). 
Figura 1. Evolución de la población mayor en España, 1949-2050

\begin{tabular}{|c|c|c|c|c|c|c|c|}
\hline \multirow{2}{*}{ A 6́os" } & \multirow{2}{*}{$\begin{array}{c}\text { Total Espania } \\
\text { Absolute }\end{array}$} & \multicolumn{2}{|c|}{$65 \mathrm{y}$ mis } & \multicolumn{2}{|c|}{75 y mis } & \multicolumn{2}{|c|}{80 y mis } \\
\hline & & Absolute & $\%$ & Absolute & $\%$ & Absolute & $\%$ \\
\hline 1900 & $18,618.086$ & 967.754 & 5,2 & 264.023 & 1,4 & 115,365 & 0,6 \\
\hline 1910 & 19.995 .686 & 1.105 .569 & 5.5 & 292.062 & 1.5 & 132.615 & 0.7 \\
\hline 1920 & 21.389 .842 & 1.216 .693 & 5.7 & 329.196 & 1.5 & 143.014 & 0.7 \\
\hline 1930 & 23.677 .794 & 1.440 .739 & 6,1 & 411.330 & 1,7 & 177.113 & 0.7 \\
\hline 1940 & 25.877 .971 & 1.690 .388 & 6,5 & 512.902 & 2,0 & 222.498 & 09 \\
\hline 1950 & 27.976 .755 & 2.022 .523 & 7,2 & 629.186 & 2,2 & 272.478 & 1,0 \\
\hline 1960 & 30.528 .539 & 2.505 .165 & 8,2 & 815.433 & 2,7 & 368.975 & 1.2 \\
\hline 1970 & 34.040 .657 & 3.290 .673 & 9.7 & 1.109 .128 & 3,3 & 523.656 & 15 \\
\hline 1981 & 37.683 .363 & 4.236 .724 & 11,2 & 1.577 .311 & 4,2 & 725.131 & 19 \\
\hline 2001 & 41.116 .842 & 7.037 .553 & 17,1 & 5.404 .513 & 13,1 & 1.663 .040 & 40 \\
\hline 2005 & 44.108 .530 & 7.332 .267 & 16,6 & 5429.048 & 12,3 & 1.903 .219 & 43 \\
\hline 2010 & 46.017 .560 & 7.742 .903 & 16,8 & 3.942 .861 & 8,6 & 2.236 .565 & 49 \\
\hline 2020 & 47.037 .942 & 9.062 .634 & 19.3 & 4.444.087 & 9,4 & 2.755 .945 & 59 \\
\hline 2030 & 47.559 .208 & 11.192 .700 & 23.5 & 5.440 .808 & 11,4 & 3.338.953 & 7,0 \\
\hline 2040 & 47.932 .948 & 13.766 .839 & 28,7 & 7.101 .429 & 14.8 & 4.366 .021 & 9,1 \\
\hline 2050 & 47.966 .653 & 15.325 .273 & 31,9 & 8.729 .934 & 18,2 & 5.644 .340 & 118 \\
\hline $\begin{array}{l}\text { Fivenur: } \\
\text { peocedi } \\
\text { INE C } \\
\text { INE I } \\
\text { INE: I }\end{array}$ & 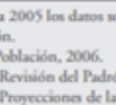 & $9=2069$ & & At & & & \\
\hline
\end{tabular}

Fuente: Abades y Rayón, 2012.

Más recientemente, los datos estadísticos del Padrón Continuo (INE) indican que a 1 de enero de 2019 hay 9.057.193 personas mayores, un 19,3\% sobre el total de la población (47.026.208) y siguen aumentando tanto en número como en proporción (Figura 2). La edad media de la población, que es otra forma de medir este proceso, se sitúa en 43,3 años; en 1970 era de 32,7 (Pérez et al., 2020).

Figura 2. Evolución de la población de 65 y más años. España, 1900-2068

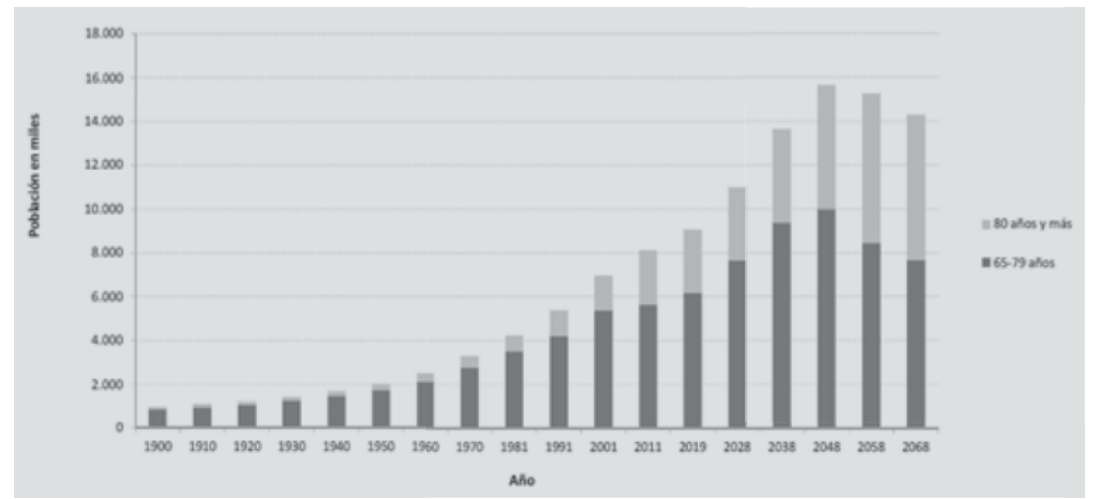

Nota: de 1900 a 2018 los datos son reales; de 2028 a 2068 se trata de proyecciones. INE:1900-2011: Censos de Población y Vivienda. 2019: Estadística del Padrón Continuo a 1-1-2019. Consulta enero 2020; 2028-2068: Proyecciones de población. Consulta enero 2019. Fuente: Pérez et al., 2020.

Estos datos hacen ver que el envejecimiento es un reto de nuestra sociedad y se debe trabajar por mejorar la calidad de vida de las personas mayores implementando programas de intervención.

El marco de la Agenda 2030 señala que las personas que están en el centro del desarrollo sostenible y la población que más crece en todo el mundo es el de las personas mayores. Por eso, se debe garantizar su bienestar y proteger sus derechos, su seguridad económica, el acceso a la atención sanitaria, a las redes de apoyo formal e informal y al aprendizaje permanente (Baviera Puig, 2020; Europe, 2019; Nations United, 2019). 
En el campo de las investigaciones relacionadas con las personas mayores se ha visto que los programas de intervención no sólo son necesarios, sino que han mostrado su efectividad. Las herramientas psicológicas contribuyen al desarrollo y puesta en práctica de sistemas de evaluación y programas de intervención para hacer frente a los problemas comportamentales y psicológicos de estas (Colegio Oficial de Psicólogos, 2002). Por tanto, el/la psicólogo/a es el/la profesional idóneo/a, dotado/a del conocimiento y habilidades necesarias para la elaboración de programas de intervención (Rodríguez et al., 2010). Uribe et al. (2004), indican que en mayores institucionalizados se debe hacer una intervención psicológica cognitivo-conductual en la que se trabajen áreas como autoestima, resignificación de la vejez, habilidades sociales, manejo del tiempo libre y autocuidado considerando que éstas contribuyen en la mejora de la misma. Por su parte, Mora et al., (2004), muestran que la calidad de vida está relacionada con variables como soporte social, autonomía, salud mental, actividad físico recreativa y la percepción subjetiva de calidad de vida; así como también con los niveles de depresión. Entonces se puede ver que la calidad de vida se ha convertido en un área de especial interés para las aplicaciones prácticas y la investigación (Verdugo et al., 2013).

Las personas mayores son un grupo vulnerable que sufre las consecuencias directas de la pandemia en su calidad de vida, enfrentando desafíos respecto al cumplimiento de sus derechos (CEPAL, 2020). En estos tiempos de COVID-19, y en especial en la etapa de confinamiento, las condiciones ambientales resultan menos estimulantes. Aumentando aún más el riesgo de padecer alguna enfermedad que repercuta en el estado de cognición de las personas mayores (Pascual et al., 1998). De ahí que en este programa se proponga una intervención basada en la promoción del autocuidado y de la activación psicológica.

El autocuidado se refiere a las distintas actividades de la vida cotidiana que son realizadas por las personas, familias y los grupos sociales para el cuidado de la salud, la prevención de enfermedades y limitación de daños (Arenas-Monreal et al., 2011).

El incremento del número de personas mayores requiere que aumente la efectividad en las formas de cuidado de la salud dirigidas hacia esta población (Durán, 2018; Zabalegui et al., 2006), lo que supone la potenciación del autocuidado, que redundará en la mejora de la calidad de vida de estas personas. La calidad de vida ha emergido en los últimos tiempos como un potente constructo, caracterizada por no ser independiente de los distintos contextos en los que la vida surge y a los que el sujeto pertenece (Fernández-Ballesteros et al., 1996).

Covid-19 ha planteado que la prevención implica centrar la atención en la salud como derecho fundamental y que las competencias emocionales son un aspecto importante de la ciudadanía efectiva y responsable (UribeRodríguez et al., 2006; Villagra y Rodríguez, 2020). Lo que supone que el dominio de éstas, dará lugar a una mejora en la adaptación al contexto y un afrontamiento más exitoso a las diferentes circunstancias de la vida (García-Blanc et al., 2020). Las competencias emocionales son necesarias para el afrontamiento de esta crisis sanitaria y de ahí su abordaje en este programa.

\section{OBJETIVO GENERAL}

Mejorar la calidad de vida en esta crisis sanitaria de las personas mayores a través de un programa de autocuidado

\section{OBJETIVOS ESPECÍFICOS}

1.Fomentar el autocuidado y la satisfacción vital

2.Entrenar en estrategias y habilidades de afrontamiento

3.Reducir los síntomas de ansiedad y depresión

\section{PARTICIPANTES}

Personas mayores sin o con deterioro cognitivo leve de diferentes entornos (residencial, domiciliario, centro de día...) del ámbito rural y urbano de Castilla y León. 


\section{METODOLOGÍA E INSTRUMENTOS UTILIZADOS}

En formato presencial u online implementación del programa de autocuidado y de activación psicológica: "Vacuna Emocional para la COVID-19" que consta de 10 sesiones de hora y media de duración en cada sesión con frecuencia semanal en formato individual o grupal.

En cada sesión se trabaja una pauta de autocuidado a través de un mensaje con forma de refrán que facilita la activación mnésica.

PROGRAMA "VACUNA EMOCIONAL EN LA COVID-19"

\begin{tabular}{|c|c|c|c|c|c|}
\hline Sesión & $\begin{array}{l}\text { Objetivo } \\
\text { s }\end{array}$ & $\begin{array}{l}\text { Contenido } \\
\text { s }\end{array}$ & $\begin{array}{l}\text { Actividad } \\
\text { es }\end{array}$ & $\begin{array}{l}\text { Metodologí } \\
\text { a }\end{array}$ & $\begin{array}{l}\text { Instrument } \\
\text { os }\end{array}$ \\
\hline $\begin{array}{l}1 \\
\text { "Cuídate sin mirar } \\
\text { de qué" }\end{array}$ & $\begin{array}{l}\text { Activar } \\
\text { conducta } \\
\text { s en } \\
\text { referenci } \\
\text { a al } \\
\text { autocuida } \\
\text { do } \\
\text { personal. }\end{array}$ & $\begin{array}{l}\text { Higiene } \\
\text { del sueño } \\
\text { Alimentaci } \\
\text { ón } \\
\text { Ejercicio } \\
\text { físico... }\end{array}$ & $\begin{array}{l}\text { Cuestionar } \\
\text { io sobre } \\
\text { los valores } \\
\text { y motivos } \\
\text { en } \\
\text { referencia } \\
\text { a las tareas } \\
\text { de } \\
\text { autocuidad } \\
\text { o. } \\
\text { Estableci } \\
\text { miento de } \\
\text { metas } \\
\text { específicas } \\
\text { en la } \\
\text { dirección } \\
\text { de los } \\
\text { valores y } \\
\text { motivos } \\
\text { personales }\end{array}$ & $\begin{array}{l}\text { Debate de } \\
\text { cuestiones. } \\
\text { Establecer } \\
\text { metas y } \\
\text { acciones } \\
\text { dirigidas. }\end{array}$ & $\begin{array}{l}\text { Registro de } \\
\text { acciones en } \\
\text { la dirección } \\
\text { de las metas } \\
\text { establecidas } \\
\text { en } \\
\text { referencia al } \\
\text { autocuidado } \\
\text {. }\end{array}$ \\
\hline
\end{tabular}




\begin{tabular}{|c|c|c|c|c|c|}
\hline $\begin{array}{l}2 \\
\text { "Con emoción, la } \\
\text { vida sale mejor" }\end{array}$ & $\begin{array}{l}\text { Identifica } \\
\text { r y } \\
\text { expresar } \\
\text { emocione } \\
\text { s y } \\
\text { sentimien } \\
\text { tos. }\end{array}$ & $\begin{array}{l}\text { Identificar } \\
\text { y gestionar } \\
\text { las } \\
\text { emociones } \\
\text { como base } \\
\text { del } \\
\text { bienestar } \\
\text { psicológic } \\
\text { o. }\end{array}$ & $\begin{array}{l}\text { Cuestionar } \\
\text { io sobre } \\
\text { actividade } \\
\text { s y } \\
\text { experienci } \\
\text { as que } \\
\text { influyan } \\
\text { en su } \\
\text { bienestar } \\
\text { emocional. } \\
\text { Activar } \\
\text { proyectos } \\
\text { de vida a } \\
\text { través de } \\
\text { acciones } \\
\text { comprome } \\
\text { tidas a } \\
\text { valores. } \\
\text { Analizar } \\
\text { las } \\
\text { barreras y } \\
\text { dificultade } \\
\text { s en la } \\
\text { gestión } \\
\text { emocional. }\end{array}$ & $\begin{array}{l}\begin{array}{l}\text { Debate de } \\
\text { cuestiones. }\end{array} \\
\text { Psicoeducac } \\
\text { ión en } \\
\text { referencia al } \\
\text { proyecto de } \\
\text { vida. }\end{array}$ & $\begin{array}{l}\text { Registro de } \\
\text { emociones: } \\
\text { identificació } \\
\text { n, } \\
\text { reconocimie } \\
\text { nto y } \\
\text { afrontamient } \\
\text { o. }\end{array}$ \\
\hline $\begin{array}{l}3 \\
\text { "Desde } \\
\text { amanecido, más } \\
\text { allá del ombligo" }\end{array}$ & $\begin{array}{l}\text { Buscar } \\
\text { estrategia } \\
\text { s } \\
\text { adecuada } \\
\text { s a los } \\
\text { medios y } \\
\text { al } \\
\text { entorno } \\
\text { para } \\
\text { relaciona } \\
\text { rse con } \\
\text { otras } \\
\text { personas. }\end{array}$ & $\begin{array}{l}\text { Las } \\
\text { relaciones } \\
\text { sociales } \\
\text { Beneficios } \\
\text { de } \\
\text { relacionart } \\
\text { e con otros }\end{array}$ & $\begin{array}{l}\text { Pregunta } \\
\text { ¿Qué } \\
\text { apoyos } \\
\text { sociales } \\
\text { tiene? } \\
\\
\text { Indagar } \\
\text { sobre } \\
\text { barreras y } \\
\text { posibilida } \\
\text { des }\end{array}$ & $\begin{array}{l}\text { Lluvia de } \\
\text { ideas }\end{array}$ & $\begin{array}{l}\text { Registro del } \\
\text { número de } \\
\text { contactos } \\
\text { mantenidos } \\
\text { a la semana. } \\
\\
\text { Escala de } \\
\text { satisfacción } \\
\text { del 1 al 10 } \\
\text { con cada } \\
\text { contacto. }\end{array}$ \\
\hline
\end{tabular}




\begin{tabular}{|c|c|c|c|c|c|}
\hline $\begin{array}{l}4 \\
\text { "El saber Sí ocupa } \\
\text { lugar” }\end{array}$ & $\begin{array}{l}\text { Promove } \\
\text { r el uso } \\
\text { adecuado } \\
\text { de la } \\
\text { informaci } \\
\text { ón y de } \\
\text { las TIC }\end{array}$ & $\begin{array}{l}\text { Infodemia } \\
\text { Saturación } \\
\text { o quemado } \\
\text { Noticias } \\
\text { falsas } \\
\text { Uso } \\
\text { adecuado } \\
\text { de internet } \\
\text { y redes } \\
\text { sociales } \\
\text { Cibersegur } \\
\text { idad }\end{array}$ & $\begin{array}{l}\text { Presentaci } \\
\text { ón de } \\
\text { informació } \\
\mathrm{n} \\
\text { Debate de } \\
\text { preguntas } \\
\text { Técnica de } \\
\text { "Saturar y } \\
\text { agrupar" }\end{array}$ & $\begin{array}{l}\text { Expositiva y } \\
\text { de diálogo } \\
\text { con una } \\
\text { dinámica } \\
\text { activa- } \\
\text { participativa }\end{array}$ & Registros \\
\hline $\begin{array}{l}5 \\
\text { "Si suena la } \\
\text { Macarena, que se } \\
\text { muevan los pies } \\
\text { sobre la arena" }\end{array}$ & $\begin{array}{l}\text { Realizar } \\
\text { la } \\
\text { activació } \\
\mathrm{n} \\
\text { conductu } \\
\text { al a } \\
\text { través de } \\
\text { la música } \\
\text { y el baile }\end{array}$ & $\begin{array}{l}\text { Relevancia } \\
\text { del } \\
\text { proyecto } \\
\text { de vida } \\
\text { atendiendo } \\
\text { a lo que es } \\
\text { valioso } \\
\text { para cada } \\
\text { persona. } \\
\text { Psicoeduc } \\
\text { ación y } \\
\text { reflexión } \\
\text { sobre la } \\
\text { relación } \\
\text { entre } \\
\text { emociones } \\
\text { y } \\
\text { conducta. } \\
\text { Importanci } \\
\text { a del valor } \\
\text { de la } \\
\text { música en } \\
\text { la } \\
\text { expresión } \\
\text { y gestión } \\
\text { emocional }\end{array}$ & $\begin{array}{l}\text { Identificac } \\
\text { ión del } \\
\text { valor que } \\
\text { tiene la } \\
\text { música y } \\
\text { el baile } \\
\text { Recordar } \\
\text { la canción } \\
\text { que te } \\
\text { guste y la } \\
\text { emoción } \\
\text { que } \\
\text { despierta. } \\
\text { Elegir una } \\
\text { canción } \\
\text { cada día } \\
\text { una } \\
\text { canción } \\
\text { para } \\
\text { escucharla } \\
\text { y/o bailar } \\
\text { que } \\
\text { evoque } \\
\text { recuerdos } \\
\text { importante } \\
\text { s en su } \\
\text { vida. }\end{array}$ & $\begin{array}{l}\text { Preguntas } \\
\text { Escritura } \\
\text { Técnicas } \\
\text { audiovisuale } \\
\mathrm{S}\end{array}$ & $\begin{array}{l}\text { Registro de } \\
\text { la canción } \\
\text { elegida, el } \\
\text { recuerdo y } \\
\text { las } \\
\text { emociones } \\
\text { que has } \\
\text { sentido al } \\
\text { escucharla. }\end{array}$ \\
\hline
\end{tabular}




\begin{tabular}{|c|c|c|c|c|c|}
\hline $\begin{array}{l}6 \\
\text { "Más allá de } \\
\text { otoño, tendrás que } \\
\text { cuidar del moño". }\end{array}$ & $\begin{array}{l}\text { Cuidar } \\
\text { de la } \\
\text { autoimag } \\
\text { en, } \\
\text { autoestim } \\
\text { a, } \\
\text { sociabilid } \\
\text { ad y } \\
\text { fomentar } \\
\text { la } \\
\text { planifica } \\
\text { ción }\end{array}$ & $\begin{array}{l}\text { Orientació } \\
\text { n a la } \\
\text { realidad. } \\
\text { Toma de } \\
\text { decisiones } \\
\text { en base a } \\
\text { la elección } \\
\text { de su ropa } \\
\text { diaria. } \\
\text { Organizaci } \\
\text { ón y } \\
\text { Planificaci } \\
\text { ón. }\end{array}$ & $\begin{array}{l}\text { Realizar la } \\
\text { planificaci } \\
\text { ón } \\
\text { semanal } \\
\text { de la ropa } \\
\text { que se va a } \\
\text { utilizar en } \\
\text { base a las } \\
\text { actividade } \\
\text { s } \\
\text { periódicas, } \\
\text { así como } \\
\text { especiales. } \\
\text { Analizar } \\
\text { gustos y } \\
\text { preferenci } \\
\text { as en base } \\
\text { a su forma } \\
\text { de vestir. }\end{array}$ & $\begin{array}{l}\text { Cuestionario } \\
\text { previo en } \\
\text { base a } \\
\text { gustos y } \\
\text { preferencias } \\
\text { en } \\
\text { referencia a } \\
\text { la ropa. } \\
\text { Registro y } \\
\text { calendario } \\
\text { semanal } \\
\text { donde figura } \\
\text { las } \\
\text { actividades } \\
\text { a realizar y } \\
\text { la ropa que } \\
\text { va a llevar } \\
\text { cada día. }\end{array}$ & $\begin{array}{l}\text { Registro y } \\
\text { calendario } \\
\text { semanal } \\
\text { donde figura } \\
\text { las } \\
\text { actividades } \\
\text { a realizar y } \\
\text { la ropa que } \\
\text { va a llevar } \\
\text { cada día. }\end{array}$ \\
\hline $\begin{array}{l}7 \\
\text { "La vida, vivida y } \\
\text { la noche, } \\
\text { dormida" }\end{array}$ & $\begin{array}{l}\text { Fomentar } \\
\text { la } \\
\text { organizac } \\
\text { ión del } \\
\text { día a día, } \\
\text { diferenci } \\
\text { ando } \\
\text { tiempo } \\
\text { de } \\
\text { actividad } \\
\text { y tiempo } \\
\text { de } \\
\text { descanso. }\end{array}$ & $\begin{array}{l}\text { - La } \\
\text { higiene del } \\
\text { sueño } \\
\text { - La } \\
\text { atención } \\
\text { plena }\end{array}$ & $\begin{array}{l}\text { Psicoeduc } \\
\text { ación } \\
\text { sobre lo } \\
\text { que es la } \\
\text { higiene del } \\
\text { sueño. } \\
\text { Actividade } \\
\text { s que } \\
\text { propician } \\
\text { la } \\
\text { excitación } \\
\text { y la } \\
\text { relajación } \\
\text { a la hora } \\
\text { de } \\
\text { conciliar } \\
\text { el sueño. } \\
\text { Identificac } \\
\text { ión de } \\
\text { pautas que } \\
\text { individual } \\
\text { mente } \\
\text { pueden } \\
\text { servirles y } \\
\text { establecer } \\
\text { los propios } \\
\text { tiempos de } \\
\text { descanso. } \\
\text { Entrenami } \\
\text { ento en } \\
\text { relajación. }\end{array}$ & $\begin{array}{l}\text { Preguntas } \\
\text { Trabajo en } \\
\text { equipo y } \\
\text { debate } \\
\text { Reflexión } \\
\text { individual } \\
\text { para llevar } \\
\text { el } \\
\text { aprendizaje } \\
\text { al día a día } \\
\text { Relajación }\end{array}$ & $\begin{array}{l}\text { Auto- } \\
\text { registro de } \\
\text { tiempos de } \\
\text { descanso y } \\
\text { calidad del } \\
\text { sueño. }\end{array}$ \\
\hline
\end{tabular}




\begin{tabular}{|c|c|c|c|c|c|}
\hline $\begin{array}{l}8 . \\
\text { "En la casa del } \\
\text { humano, a utilizar } \\
\text { la mano" }\end{array}$ & $\begin{array}{l}\text { Realizar } \\
\text { la } \\
\text { activació } \\
\mathrm{n} \\
\text { conductu } \\
\text { al a } \\
\text { través de } \\
\text { la } \\
\text { práctica } \\
\text { de } \\
\text { aficiones } \\
\text { y } \\
\text { hobbies. }\end{array}$ & $\begin{array}{l}\text { Relevancia } \\
\text { del } \\
\text { proyecto } \\
\text { de vida } \\
\text { atendiendo } \\
\text { a lo que es } \\
\text { valioso } \\
\text { para cada } \\
\text { persona. } \\
\text { Psicoeduc } \\
\text { ación y } \\
\text { reflexión } \\
\text { sobre la } \\
\text { relación } \\
\text { entre } \\
\text { emociones } \\
\text { y } \\
\text { conducta. } \\
\text { Importanci } \\
\text { a de la } \\
\text { activación } \\
\text { de hobbies } \\
\text { y aficiones } \\
\text { para vivir } \\
\text { una vida } \\
\text { con } \\
\text { sentido } \\
\text { con } \\
\text { bienestar } \\
\text { emocional. }\end{array}$ & $\begin{array}{l}\text { Identificac } \\
\text { ión y } \\
\text { registro de } \\
\text { las } \\
\text { actividade } \\
\text { s que se } \\
\text { están } \\
\text { llevando a } \\
\text { cabo, las } \\
\text { que se han } \\
\text { abandonad } \\
\text { o, las } \\
\text { rutinas } \\
\text { perdidas y } \\
\text { el papel de } \\
\text { familiares } \\
\text { y amigos } \\
\text { cómo } \\
\text { reforzador } \\
\text { es o } \\
\text { inhibidore } \\
\text { s de las } \\
\text { acciones. } \\
\\
\text { Elaboració } \\
\text { n del } \\
\text { listado de } \\
\text { actividade } \\
\text { s a realizar } \\
\text { en base a } \\
\text { una } \\
\text { jerarquía. } \\
\text { Selección } \\
\text { de una } \\
\text { actividad } \\
\text { que hace } \\
\text { tiempo } \\
\text { que no se } \\
\text { practica y } \\
\text { llevarla a } \\
\text { cabo } \\
\text { registrand } \\
\text { o las } \\
\text { emociones } \\
\text { elicitadas. }\end{array}$ & $\begin{array}{l}\text { Debate } \\
\text { Técnicas } \\
\text { audiovisuale } \\
\text { s } \\
\text { Metáforas y } \\
\text { ejercicios } \\
\text { para la } \\
\text { clarificación } \\
\text { de lo } \\
\text { importante }\end{array}$ & 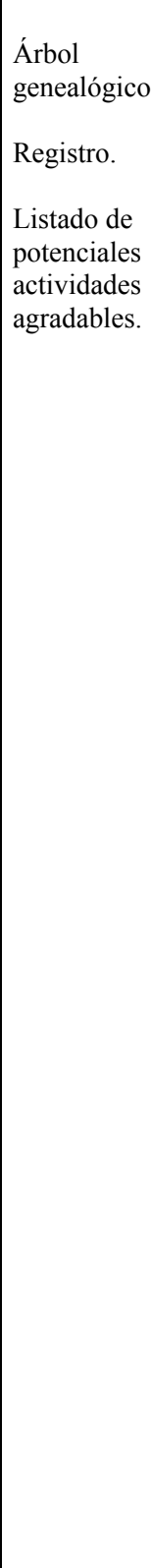 \\
\hline
\end{tabular}




\begin{tabular}{|c|c|c|c|c|c|}
\hline $\begin{array}{l}9 \\
\text { "Las memorias } \\
\text { recordadas, de } \\
\text { nuevo van a ser } \\
\text { guardadas" }\end{array}$ & $\begin{array}{l}\text { Preservar } \\
\text { y } \\
\text { reforzar } \\
\text { la } \\
\text { identidad } \\
\text { personal } \\
\text { y el } \\
\text { sentido } \\
\text { de } \\
\text { continuid } \\
\text { ad vital }\end{array}$ & $\begin{array}{l}\text { Memoria } \\
\text { autobiográ } \\
\text { fica. } \\
\text { Contexto } \\
\text { histórico } \\
\text { social - } \\
\text { cultural y } \\
\text { familiar. } \\
\text { Revisión } \\
\text { de vida. } \\
\text { Emociones } \\
\text { vinculadas } \\
\text { Integració } \\
\text { n del } \\
\text { pasado con } \\
\text { el } \\
\text { presente. } \\
\text { Identidad } \\
\text { personal } \\
\text { Estrategias } \\
\text { de } \\
\text { Afrontami } \\
\text { ento. }\end{array}$ & $\begin{array}{l}\text { Evocación } \\
\text { de } \\
\text { situacione } \\
\text { s del } \\
\text { pasado } \\
\text { (Etapas } \\
\text { vitales) } \\
\text { Recuperac } \\
\text { ión de } \\
\text { recuerdos } \\
\text { autobiográ } \\
\text { ficos } \\
\text { significati } \\
\text { vos } \\
\text { Retrato } \\
\text { personal: } \\
\text { Auto- } \\
\text { reconocim } \\
\text { iento } \\
\text { Listado de } \\
\text { competenc } \\
\text { ias y } \\
\text { habilidade } \\
\text { s }\end{array}$ & $\begin{array}{l}\text { Debate } \\
\text { Escritura } \\
\text { Creativa } \\
\text { Dibujo, } \\
\text { pintura y } \\
\text { Collage } \\
\text { Ejercicios } \\
\text { estimulación } \\
\text { cognitiva y } \\
\text { de } \\
\text { reminiscenci } \\
\text { a } \\
\text { Técnicas } \\
\text { audiovisuale } \\
\text { s }\end{array}$ & $\begin{array}{l}\begin{array}{l}\text { Árbol } \\
\text { genealógico }\end{array} \\
\text { Libro de } \\
\text { vida } \\
\text { Baúl de } \\
\text { recuerdos } \\
\text { Inventario } \\
\text { de } \\
\text { competencia } \\
\text { s } \\
\text { individuales } \\
\text { y grupales } \\
\text { Guión de } \\
\text { proyecto de } \\
\text { vida: Ruta } \\
\text { vital y } \\
\text { valores guía. }\end{array}$ \\
\hline $\begin{array}{l}10 \\
\text { "Como el mus, } \\
\text { órdago a la vida" }\end{array}$ & $\begin{array}{l}\text { Conectar } \\
\text { con los } \\
\text { valores } \\
\text { que guían } \\
\text { y dan } \\
\text { sentido a } \\
\text { la vida }\end{array}$ & $\begin{array}{l}\text { Psicoeduc } \\
\text { ación } \\
\text { desde la } \\
\text { ACT: } \\
\text { volver a } \\
\text { vivir una } \\
\text { vida que } \\
\text { merezca la } \\
\text { pena } \\
\text { reexperim } \\
\text { entando el } \\
\text { contacto } \\
\text { con las } \\
\text { fuentes de } \\
\text { recompens } \\
\text { a de la vida } \\
\text { (activación } \\
\text { de } \\
\text { proyectos } \\
\text { vitales). } \\
\text { Áreas de } \\
\text { Valor, } \\
\text { pasiones y } \\
\text { talentos }\end{array}$ & $\begin{array}{l}\text { Preguntas } \\
\text { para hablar } \\
\text { de lo } \\
\text { importante } \\
\text { Metáfora } \\
\text { del Jardín. } \\
\text { Identficaci } \\
\text { ón de } \\
\text { valores en } \\
\text { cada área } \\
\text { vital } \\
\text { valiosa } \\
\text { para la } \\
\text { persona. } \\
\text { Selección } \\
\text { de un } \\
\text { proyecto y } \\
\text { llevarlo a } \\
\text { cabo. }\end{array}$ & $\begin{array}{l}\text { Debate } \\
\text { Técnicas } \\
\text { audiovisuale } \\
\text { s } \\
\text { Metáforas y } \\
\text { ejercicios } \\
\text { para la la } \\
\text { clarificación } \\
\text { de lo } \\
\text { importante }\end{array}$ & $\begin{array}{l}\text { Cuestionario } \\
\text { s de Valores. } \\
\text { Listados de } \\
\text { Areas } \\
\text { Vitales. } \\
\text { Formulario } \\
\text { de acciones, } \\
\text { objetivos y } \\
\text { barreras. }\end{array}$ \\
\hline
\end{tabular}


Utilizando un diseño cuasiexperimental pre-post con grupo control, se emplean los instrumentos:

-Escala FUMAT de calidad de vida (Verdugo et al., 2009). Está compuesta por un total de 80 ítems, enunciados en tercera persona, con formato declarativo, y ordenados de forma aleatoria. Todos los ítems pueden responderse con un formato de respuesta de frecuencia de cuatro opciones ('nunca o casi nunca', 'a veces', 'frecuentemente', 'siempre o casi siempre'). Posee un índice de fiabilidad de .962.

- Escala de Satisfacción con la Vida (Pons et al. 2002). Es una escala de 5 ítems que evalúa el juicio global que hacen las personas sobre la satisfacción con su vida. El índice de fiabilidad calculado para la escala alpha de Cronbach, indica que la escala tiene una muy buena consistencia interna $(=.84)$.

- Acceptance and Action Questionnaire-II (Barraca, 2004). Es una escala que consta de 9 ítems en una escala tipo likert (1-7) en los que se evalúa la evitación experiencial y la aceptación psicológica. La consistencia interna es de .74 .

- Escala de Depresión Geriátrica de Yesavage (Martínez et al., 2002). Es un instrumento ampliamente utilizado para la evaluación de la depresión. Una puntuación de 50 más respuestas positivas sugiere depresión, con una sensibilidad y especificidad de entre 80-95\% dependiendo de la población estudiada.

- Inventario de Ansiedad de Beck (Sanz et al., 2014). Es una de las escalas más utilizadas para la evaluación de la ansiedad. Consta de 21 ítems en una escala tipo Likert (1-4). El estudio de la fiabilidad de la adaptación española se restringe a la consistencia interna, produciendo en diversas muestras un valor del coeficiente alfa alto (superior a 0.8)

\section{RESULTADOS ESPERADOS}

La reducción de la sintomatología ansiosa y/o depresiva; Mejora en la calidad de vida, concretamente en bienestar físico, bienestar emocional, relaciones interpersonales, inclusión social, desarrollo personal, bienestar material, autodeterminación y derechos; Aumento de la satisfacción con la vida, en la muestra diana respecto al grupo control.

\section{CONCLUSIONES}

La necesidad de este tipo de intervención psicológica en el ámbito gerontológico destaca por su viabilidad, eficacia y demanda social. Este programa se centra en el proceso de envejecimiento individual y centrado en la persona y alejado de una visión homogénea de este colectivo. Se persigue una intervención integral que tenga en cuenta aspectos físicos, psicológicos, sociales y espirituales. Por otro lado, también se centra en fomentar la capacidad de autodeterminación en la vida, la toma de decisiones y la visibilización de competencias y fortalezas. En la línea de Uribe et al. (2004), este programa constituye una intervención psicológica cognitivo-conductual en el que se trabajan distintos aspectos que contribuyen a la mejora de la calidad de vida apoyándose en el marco teórico de la Terapia de Aceptación y Compromiso y utilizando la activación conductual, una estrategia que potencia el afrontamiento de situaciones estresantes como la crisis sanitaria actual por la COVID-19 y refuerza la continuidad del ciclo vital.

\section{REFERENCIAS BIBLIOGRÁFICAS}

Abades Porcel, M., \& Rayón Valpuesta, E. (2012). El envejecimiento en España: ¿un reto o problema social? Gerokomos, 23 (4), 151-155 https://dx.doi.org/10.4321/S1134-928X2012000400002

Abellán, A., Aceituno, P., Pérez, J., Ramiro, D., Ayala, A., y Pujol, R. (2019). Un perfil de las personas mayores en España, 2019 indicadores estadísticos básicos.

Arenas-Monreal, L., Jasso-Arenas, J., \& Campos-Navarro, y R. (2011). Autocuidado: elementos para sus bases conceptuales. Global Health Promotion, 18(4), 42-48. https://doi.org/10.1177/1757975911422960

Barraca, J. (2004). Spanish adaptation of the Acceptande and Action Questionnarie (AAQ). International Journal of Psychology and Psychological Therapy, 4(3), 505-515. 
Baviera Puig, I. (2020). Desarrollo sostenible, transformación digital y atención a las personas: oportunidades y riesgos del envejecimiento demográfico. Relaciones Laborales y Derecho del Empleo, 8(1).

Caribe, Comisión Económica para América Latina y el. América Latina y el Caribe ante la pandemia del COVID19: efectos económicos y sociales. CEPAL, 2020. https://www.cepal.org/es/publicaciones/45337-americalatina-caribe-la-pandemia- covid-19-efectos-economicos-sociales

Colegio Oficial de Psicólogos (2002). Psicología, psicólogos y envejecimiento: Contribución de la psicología y Ios psicólogos al estudio y la intervención sobre el envejecimiento. Revista Especializada en Gerontología, 37 (5), 268-275.

Durán, M. Á. (2018). La riqueza invisible del cuidado (Vol. 30). Universitat de València.

Europe, A. (2019). Looking at the lives of older people in the EU. 2019 edition. Luxembourg: Publications Office of the European Union, 157.

Fernández-Ballesteros, R., Zamarrón, M.D. y Maciá, A. (1996). Calidad de vida en distintos contextos en la vejez. Madrid: Instituto Nacional de Servicios Sociales (INSERSO).

García-Blanc, N., Gomis, R., Ros Morente, A., y Filella Guiu, G. (2020). El proceso de gestión emocional: investigación, aplicación y evaluación. Know and Share Psychology, 2020, vol. 1, núm. 4, p. 39-49. http://dx.doi.org/10.25115/kasp.v1i4.4260

Imserso (2009). Indicadores demográficos. Las personas mayores en España. Informe 2008. Tomo 1. 1 ed. Madrid: Instituto de Mayores y Servicios Sociales.

Lorenzo, L. (2004). Consecuencias del envejecimiento de la población: el futuro de las pensiones. Indicadores sociales de España, 2004, 73-92. http://www.ine.es/ prodyser/pubweb/indisoc04/indisoc04.htm

Martínez, J., Onís, M. C., Dueñas, R., Albert, C., Aguado, C., \& Luque, R. (2002). Versión española del cuestionario de Yesavage abreviado (GDS) para el despistaje de depresión en mayores de 65 años: adaptación y validación. Medifam, 12(10), 620-630.

Mora, M., Villalobos, D., Araya, G., y Ozols, A. (2004). Perspectiva subjetiva de la calidad de vida del adulto mayor, diferencias ligadas al género ya la práctica de la actividad físico recreativa. MHSALUD: Revista en Ciencias del Movimiento Humano y Salud, 1(1). https://doi.org/10.15359/mhs.1-1.3

Nations United. (2019). United Nations. World Population Prospects 2019: Highlights. Statistical Papers-United Nations (Ser. A), Population and Vital Statistics Report.

Pascual, G., Barlés, G., Laborda Soriano, A., \& Loren Torres, L. (1998). Cognición-ocupación: Un binomio unido en la vejez. Revista Geriátrica, 1998; 14 (3): 57, 60.

Pérez, J.; Abellán, A.; Aceituno, P.; Ramiro, D. (2020). "Un perfil de las personas mayores en España, 2020. Indicadores estadísticos básicos". Madrid, Informes Envejecimiento en red no 25, 39 pp. 5 http://envejecimiento.csic.es/documentos/documentos/enred-indicadoresbasicos2020.pdf>

Pons, D., Atienza, F. L., Balaguer, I., y García-Merita, M. (2002). Propiedades psicométricas de la escala de satisfacción con la vida en personas de tercera edad. Revista Iberoamericana de Diagnóstico y Evaluación Psicológica.

Rodríguez, A.F., Valderrama, L.J., y Molina, J.M. (2010). Intervención psicológica en adultos mayores. Psicología desde el Caribe, (25),246-258 https://www.redalyc.org/articulo.oa?id=21315106011

Rodríguez, P. (2021). Género y envejecimiento en España. Perfiles, datos y reflexiones. Papeles de la Fundación, Pilares para la Autonomía Personal, $n^{0} 6$.

Sanz, J. (2014). Recomendaciones para la utilización de la adaptación española del Inventario de Ansiedad de Beck (BAl) en la práctica clínica. Clínica y Salud, 25(1), 39-48.

Uribe, A., Padilla, C., Ramírez, V. (2004). Intervención cognitivo conductual para la calidad de vida en viejos institucionalizados. Revista Iberoamericana de Geriatría y Gerontología: Geriátrika, 20 (3), 145-149. 
Uribe-Rodríguez, A. F., Molina, J. M., \& González, L. (2006). Program of intervention on the quality of life, the depression and the cognitive deterioration in people who belong to groups of the third age. In Documento presentado en la 26th International Congress of Applied Psychology, Atenas, Grecia.

Verdugo, M. A., Schalock, R. L., Arias, B., Gómez, L., \& Jordán de Urríes, B. (2013). Calidad de vida. MA Verdugo \& RL Schalock (Coords.), Discapacidad e inclusión manual para la docencia, 443-461.

Verdugo Alonso, M. Á., Gómez Sánchez, L. E., y Arias Martínez, B. (2009). Evaluación de la calidad de vida en personas mayores: La escala FUMAT. Instituto Universitario de Integración en la Comunidad (Salamanca, España).

Villagra, G. S., \& Rodríguez, A. C. (2020). Estrategias de afrontamiento en tiempo de coronavirus. http://hdl.handle.net/11336/111813

Zabalegui, A., Escobar, M. A., Cabrera, E., Gual, M. P., Fortuny, M., Mach, G., Ginesti, M. \& Narbona, P. (2006). Análisis del programa educativo PECA para mejorar la calidad de vida de las personas mayores. Atención Primaria, 37 (5), 260-265. https://doi.org/10.1157/13086310 
